\title{
The Projections of Convex Lattice Sets of Points in $\mathbb{E}^{2}$
}

\author{
Yu Gu and Lin Si \\ College of Science, Beijing Forestry University, Beijing 100083, China \\ Correspondence should be addressed to Lin Si; silin@bjfu.edu.cn \\ Received 10 May 2016; Revised 17 October 2016; Accepted 25 October 2016 \\ Academic Editor: Francesco Soldovieri
}

Copyright (C) 2016 Y. Gu and L. Si. This is an open access article distributed under the Creative Commons Attribution License, which permits unrestricted use, distribution, and reproduction in any medium, provided the original work is properly cited.

\begin{abstract}
Can one determine a centrally symmetric lattice polygon by its projections? In 2005, Gardner et al. proposed the above discrete version of Aleksandrov's projection theorem. In this paper, we define a coordinate matrix for a centrally symmetric convex lattice set and suggest an algorithm to study this problem.
\end{abstract}

\section{Introduction}

According to Gardner [1], geometric tomography deals with the retrieval of information about a geometric object from data concerning its projections (shadows) on planes or cross sections by planes. When the geometric object is a convex body, there are many results from convex geometry. A famous example is the celebrated Aleksandrov's projection theorem, that is, for an origin symmetric convex body (compact, convex subset with nonempty interior in the $n$-dimensional Euclidean space $\mathbb{E}^{n}$ ); it can be determined by its projections. For more details, see [1]. When the object is a convex lattice set, it models the atoms in a crystal which will lead to a range of topics concerned with electron microscopy in discrete tomography $[2,3]$.

If $A$ is a set in $\mathbb{E}^{n}$, we denote the convex hull of $A$ by conv $A$. The dimension of $A$ is the dimension of its affine hull and is denoted by $\operatorname{dim} A$. The notation for the usual orthogonal projection of $A$ on a subspace $H$ is $A$ | $H$. A convex lattice set $F$ is a finite subset of the $n$-dimensional integer lattice $\mathbb{Z}^{n}$ such that $F=\operatorname{conv} F \cap \mathbb{Z}^{n}$ and we denote the cardinality of $F$ by $\sharp\{F\}$. Let $C\left(\mathbb{Z}^{n}\right)$ denote the set of all centrally symmetric convex lattice sets about origin of $\mathbb{Z}^{n}$.

In 2005, Gardner et al. [4] proposed the following discrete version of Aleksandrov's projection theorem.

Question 1. Let $C_{1}$ and $C_{2}$ belong to $C\left(\mathbb{Z}^{n}\right)$ with $\operatorname{dim} C_{1}=$ $\operatorname{dim} C_{2}$. If $\sharp\left\{C_{1} \mid H\right\}=\sharp\left\{C_{2} \mid H\right\}$ holds for every hyperplane $H$ with a norm $u \in \mathbb{Z}^{n} \backslash\{o\}$, do $C_{1}$ and $C_{2}$ coincide up to a translation?
In [4], Gardner et al. discovered a counterexample to this problem in $\mathbb{Z}^{2}$ with cardinality 11 (Figure 1). In [5], Zhou proved that the counterexample discovered by Gardner et al. was the only counterexample in $\mathbb{Z}^{2}$, up to unimodular transformations and with cardinality not larger than 17. In [6], Xiong proved that an origin symmetric convex lattice set can be uniquely determined by its lattice projection counts if $y$ coordinate's absolute value is not bigger than 2 for every point in this origin symmetric convex lattice set and its cardinality is not 11 .

All the results above [4-6] show that the discrete Aleksandrov's projection theorem must have additional hypotheses. Motivated by the work of Gardner, Gronchi, Zong, Zhou, and Xiong, in the present paper, we introduced the coordinate matrix for convex lattice set and present some results about coordinate matrix. Considering that counting of projection of a convex lattice set in the discrete Aleksandrov's projection theorem is important, we propose an algorithm based on the coordinate matrix and its properties.

\section{Coordinate Matrix}

Let $P$ belong to $C\left(\mathbb{Z}^{n}\right)$. If $P$ has $a_{i}$ points on the line $x=$ $i(-m \leq i \leq m)$, where $a_{m} \neq 0$ and $a_{m+1}=a_{m+2}=\cdots=0$, then we denote $P$ by $P_{\left[a_{-m}, \ldots, a_{-1}, a_{0}, a_{1}, \ldots, a_{m}\right]}$. Clearly, since $P$ is an origin symmetric convex lattice set, we have that $a_{i}=a_{-i}(1 \leq$ $i \leq m), \sharp\{P\}=\sum_{i=-m}^{m} a_{i}$.

Definition 1. For $P_{\left[a_{-m}, \ldots, a_{-1}, a_{0}, a_{1}, \ldots, a_{m}\right]}$, denote the minimum $y$ coordinate by $y_{i 0}$ and the maximum $y$-coordinate by $y_{i 1}$ on 


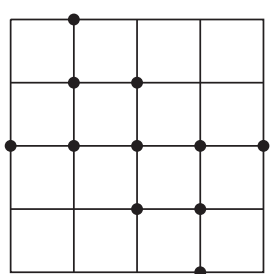

$C_{1}$
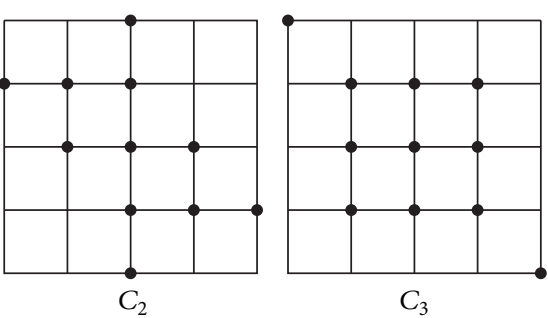

$\mathrm{C}_{3}$

FIgURE 1: Counterexample to Question 1 in $\mathbb{Z}^{2}$ with cardinality 11.

the line $x=i(-m \leq i \leq m)$, respectively. Define the coordinate matrix of $P_{\left[a_{-m}, \ldots, a_{-1}, a_{0}, a_{1}, \ldots, a_{m}\right]}$ by the following:

$$
\left(\begin{array}{cccccc}
x_{0} & x_{1} & \cdots & x_{i} & \cdots & x_{m} \\
y_{01} & y_{11} & \cdots & y_{i 1} & \cdots & y_{m 1} \\
y_{00} & y_{10} & \cdots & y_{i 0} & \cdots & y_{m 0}
\end{array}\right) .
$$

Obviously, a convex lattice set and its coordinate matrix can be uniquely determined by each other.

Example 2. Considering the coordinate matrix of $C_{1}$ in Figure 1, we get

$$
\left(\begin{array}{ccc}
0 & 1 & 2 \\
1 & 0 & 0 \\
-1 & -2 & 0
\end{array}\right)
$$

We summarize some basic properties of coordinate matrix as the following propositions.

\section{Theorem 3. Let}

$$
\begin{aligned}
P & \in C\left(\mathbb{Z}^{n}\right) \\
M & =\left(\begin{array}{llllll}
x_{0} & x_{1} & \cdots & x_{i} & \cdots & x_{m} \\
y_{01} & y_{11} & \cdots & y_{i 1} & \cdots & y_{m 1} \\
y_{00} & y_{10} & \cdots & y_{i 0} & \cdots & y_{m 0}
\end{array}\right)
\end{aligned}
$$

be its coordinate matrix. Then $M$ has the following properties:

(1) $\sharp\{P\}=2 \sum_{i=1}^{m}\left|y_{i 1}-y_{i 0}\right|+\left|y_{01}-y_{00}\right|+2 m+1$.

(2) $\sharp\{P \mid y=0\}=2 m+1$.

(3) $\sharp\{P \quad x \quad=0\}=$

Proof. Let

$$
\begin{aligned}
P & \in C\left(\mathbb{Z}^{n}\right) \\
M & =\left(\begin{array}{llllll}
x_{0} & x_{1} & \cdots & x_{i} & \cdots & x_{m} \\
y_{01} & y_{11} & \cdots & y_{i 1} & \cdots & y_{m 1} \\
y_{00} & y_{10} & \cdots & y_{i 0} & \cdots & y_{m 0}
\end{array}\right)
\end{aligned}
$$

(1) By the definition of the coordinate matrix and the symmetry of $P$, we have

$$
\begin{aligned}
\sharp\{P\} & =2\left(\sum_{i=1}^{m}\left|y_{i 1}-y_{i 0}\right|+1\right)+\left(\left|y_{01}-y_{00}\right|+1\right) \\
& =2 \sum_{i=1}^{m}\left|y_{i 1}-y_{i 0}\right|+\left|y_{01}-y_{00}\right|+2 m+1 .
\end{aligned}
$$

(2) From the the definition of the coordinate matrix $P$, we can get it.

(3) Note that $P$ is symmetric about the origin.

For $P \in C\left(\mathbb{Z}^{n}\right)$ and its coordinate matrix

$$
M=\left(\begin{array}{cccccc}
x_{0} & x_{1} & \cdots & x_{i} & \cdots & x_{m} \\
y_{01} & y_{11} & \cdots & y_{i 1} & \cdots & y_{m 1} \\
y_{00} & y_{10} & \cdots & y_{i 0} & \cdots & y_{m 0}
\end{array}\right),
$$

we can denote by $\left( \pm x_{i}, \pm y_{i r}\right),\left( \pm x_{j}, \pm y_{j s}\right)$, and $\left( \pm x_{k}, \pm y_{k t}\right)$ any three points in $P$, where $0 \leq i, j, k \leq m$, and $y_{i 0} \leq y_{i r} \leq y_{i 1}$, $y_{j 0} \leq y_{j s} \leq y_{j 1}, y_{k 0} \leq y_{k t} \leq y_{k 1}$.

Remark 4. Here $\left( \pm x_{i}, \pm y_{i r}\right),\left( \pm x_{j}, \pm y_{j s}\right)$, and $\left( \pm x_{k}, \pm y_{k t}\right)$ denote one of the following cases:

$$
\begin{aligned}
& \left((-1)^{u_{1}} x_{i},(-1)^{u_{2}} y_{i r}\right), \\
& \left((-1)^{v_{1}} x_{j},(-1)^{v_{2}} y_{j s}\right), \\
& \left((-1)^{w_{1}} x_{k},(-1)^{w_{2}} y_{k t}\right),
\end{aligned}
$$

for $u_{1}, u_{2}, v_{1}, v_{2}, w_{1}$, and $w_{2} \in\{1,2\}$.

If

$$
\left|\begin{array}{ccc} 
\pm x_{i} & \pm y_{i r} & 1 \\
\pm x_{j} & \pm y_{j s} & 1 \\
\pm x_{k} & \pm y_{k t} & 1
\end{array}\right| \neq 0
$$

we know that $\left( \pm x_{i}, \pm y_{i r}\right),\left( \pm x_{j}, \pm y_{j s}\right)$, and $\left( \pm x_{k}, \pm y_{k t}\right)$ can be regarded as three vertexes of a nondegenerate triangle.

Then, with $0 \leq \lambda_{1}, \lambda_{2}, \lambda_{3} \leq 1$ and $\lambda_{1}+\lambda_{2}+\lambda_{3}=1$, we have that

$$
\begin{aligned}
& x_{p}=\lambda_{1}\left( \pm x_{i}\right)+\lambda_{2}\left( \pm x_{j}\right)+\lambda_{3}\left( \pm x_{k}\right) \\
& y_{p}=\lambda_{1}\left( \pm y_{i r}\right)+\lambda_{2}\left( \pm y_{j s}\right)+\lambda_{3}\left( \pm y_{k t}\right)
\end{aligned}
$$

be its coordinate matrix. 


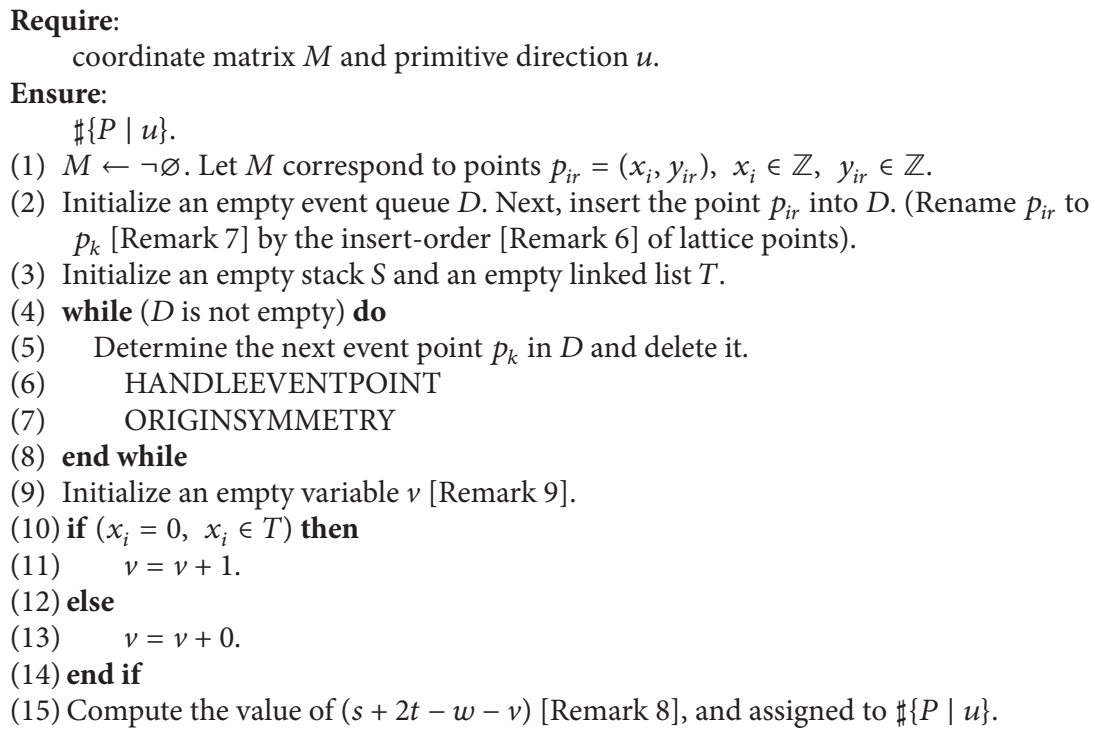

Algorithm 1: Plane sweep algorithm.

is a convex combination of $\left( \pm x_{i}, \pm y_{i r}\right),\left( \pm x_{j}, \pm y_{j s}\right)$, and $\left( \pm x_{k}, \pm y_{k t}\right)$. If $\left(x_{p}, y_{p}\right) \in \mathbb{Z}^{2}$, by the convexity of $P,\left(x_{p}, y_{p}\right)$ belongs to $P$. Thus we obtain the following theorem.

Theorem 5. Let

$$
M=\left(\begin{array}{cccccc}
x_{0} & x_{1} & \cdots & x_{i} & \cdots & x_{m} \\
y_{01} & y_{11} & \cdots & y_{i 1} & \cdots & y_{m 1} \\
y_{00} & y_{10} & \cdots & y_{i 0} & \cdots & y_{m 0}
\end{array}\right)
$$

with $x_{i}=i$ and $y_{i 1}>y_{i 0}, y_{i 1}, y_{i 0} \in \mathbb{Z}, i=0,1,2, \ldots, m$. Then $M$ is the coordinate matrix of some $P \in C\left(\mathbb{Z}^{n}\right)$ if and only iffor any $\lambda_{1}, \lambda_{2}, \lambda_{3}$ with $0 \leq \lambda_{1}, \lambda_{2}, \lambda_{3} \leq 1$ and $\lambda_{1}+\lambda_{2}+\lambda_{3}=1$ there exist $x_{q}, y_{q 1}, y_{q 0}$ in $M$ such that

$$
\begin{gathered}
x_{q}= \pm x_{p}, \\
y_{q 1} \geq \pm y_{p} \geq y_{q 0} .
\end{gathered}
$$

\section{Algorithm for Counting of Projection of a Convex Lattice Set}

Let $P \in C\left(\mathbb{Z}^{n}\right)$. The primitive directions of $P, D S(P)$ is defined as

$$
\begin{aligned}
& D S(P)=\{u=(a, b) \mid a, b \in \mathbb{Z}, \operatorname{gcd}(a, b)=1, \exists p, q \\
& \left.\quad \in P \text {, such that } l_{p q} \| u\right\} .
\end{aligned}
$$

Here, gcd represents the greatest common divisor.
Let

$$
M=\left(\begin{array}{cccccc}
x_{0} & x_{1} & \cdots & x_{i} & \cdots & x_{m} \\
y_{01} & y_{11} & \cdots & y_{i 1} & \cdots & y_{m 1} \\
y_{00} & y_{10} & \cdots & y_{i 0} & \cdots & y_{m 0}
\end{array}\right)
$$

be the coordinate matrix of $P$, and $u=(a, b) \in D S(P)$. Based on the line segment intersection algorithm [7], the computation of planar convex hulls algorithm [7], and the coordinate matrix of $P$, an algorithm named as plane sweep algorithm about $\sharp\{P \mid u\}, \forall u \in D S(P)$ is given in Algorithm 1 .

Remark 6. Moving a horizontal sweep line $l$ downwards over the plane, the sweep line halts at certain event points. If sweep line reaches an event point, then a lattice point must be added to event queue $D$. This point is tested against the ones already have been reached. If two points have the same $y$-coordinate, then the one with smaller $x$-coordinate will be added to event queue $D$ first.

Remark 7. Based on Theorem $3, M$ is a $3 \times(m+1)$ matrix and it presents $\sum_{i=0}^{m}\left(y_{i 1}-y_{i 0}+1\right)$ points. We denote $\sum_{i=0}^{m}\left(y_{i 1}-y_{i 0}+1\right)$ by $N$. Thus $D$ could be recorded as $\left\{p_{k}, 1 \leqslant k \leqslant N\right\}$.

Remark 8. $s, t$, and $w$ are obtained after the While-loop is completed. At the end of Algorithm 2,s and $t$ represent the number of points in $S$ and T, respectively. $w$ is defined in Algorithm 3.

Remark 9. The points on the $y$-axis should be considered separately because their symmetric points about the origin are still on the $y$-axis. The If-loop at the end of Algorithm 1 is used to do this. $v$ represents the number of points whose $x$-coordinates are zero in $T$. 


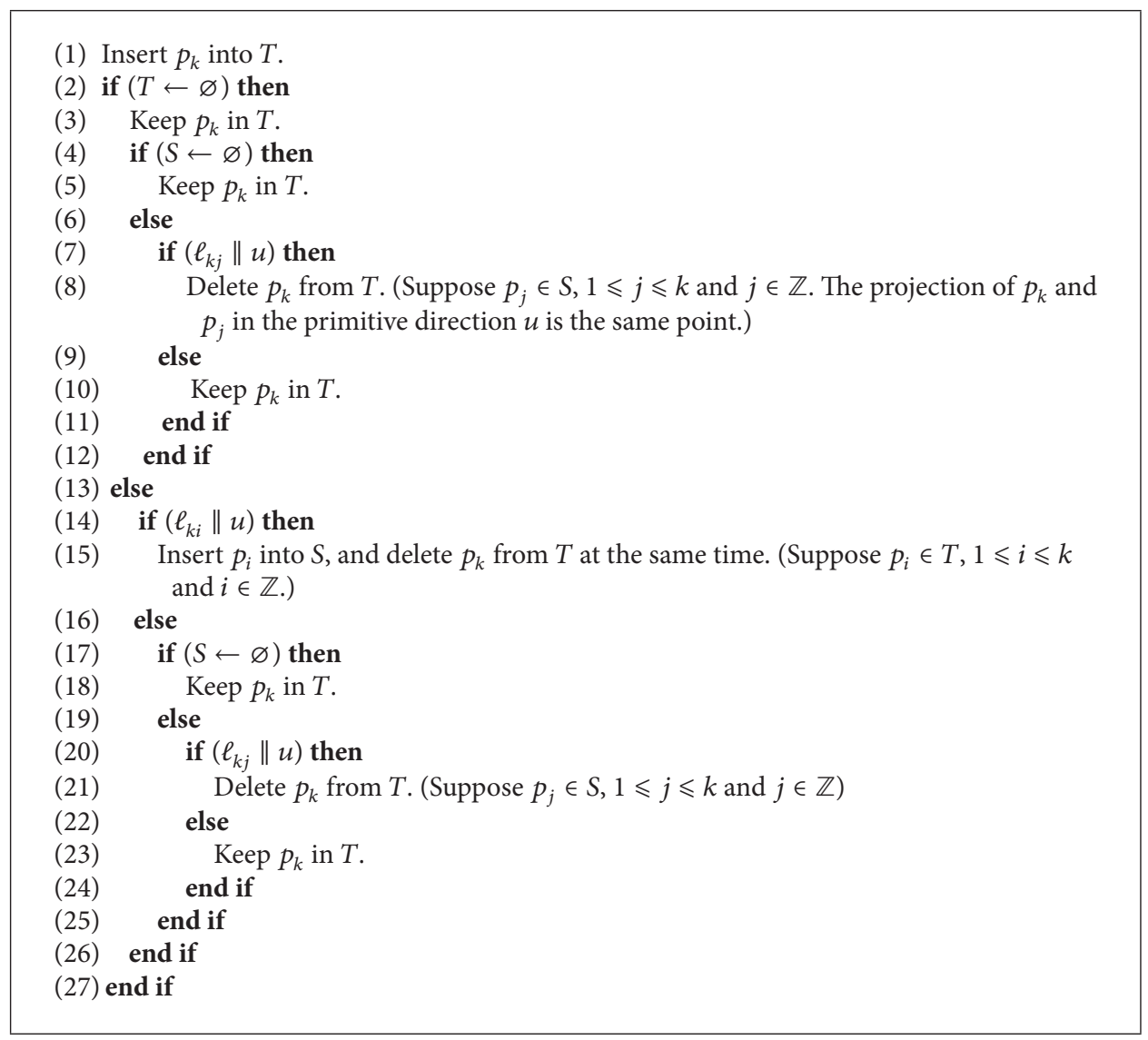

Algorithm 2: HANDLEEVENTPOINT.

(1) The origin symmetric point of $p_{i}$ except those on the $y$-coordinate is denoted by $q_{i}$.

(2) $\ell_{q_{i} p_{i}}$ should be used to express the line though $p_{i} \in T$ and $q_{i}$.

(3) Initialize an empty variable $w$.

(4) if $\left(\ell_{q_{i} p_{i}} \| u\right)$ then

(5) $w=w+1$.

(6) else

(7) $\quad w=w+0$.

(8) end if

Algorithm 3: ORIGINSYMMETRY.

The procedures for finding the lattice points of the projection overlapped as follows: we simply test if the direction of a straight line that is determined by two lattice points is parallel to the primitive directions. We could delete the lattice points which conform to the conditions in order to simplify the computation. Hence, the procedure HANDLEEVENTPOINT is defined as in Algorithm 2.

By the origin symmetry, we have less calculation of nearly half of the points. The next procedure ORIGINSYMMETRY, defined as in Algorithm 3, is used to handle the missing points which are caused by the origin symmetry. In this procedure the way for process the lattice points whose projection will be overlap is the same as the procedure HANDLEEVENTPOINT.

Remark 10. If we do not consider the origin symmetry, we should calculate $2 N-1-y_{01}+y_{00}$ points. This will take $\mathrm{O}\left(\left(2 N-1-y_{01}+y_{00}\right) \times\left(2 N-2-y_{01}+y_{00}\right)\right)$ time.

The time required for the parts of HANDLEEVENTPOINT and ORIGINSYMMETRY dominates the total running time. In HANDLEEVENTPOINT, considering the worst case, we check every point with other $N-1$ points whether they are in the same straight line with primitive 
direction. This will take $O((1 / 2) N \times(N-1))$ time. In ORIGINSYMMETRY, we check $N$ points with other $N$ $\left(y_{01}-y_{00}+1\right)$ points. This will take $O((1 / 2) N \times(N-1-$ $\left.\left.y_{01}+y_{00}\right)\right)$ time.

\section{Competing Interests}

The authors declare that they have no competing interests.

\section{Acknowledgments}

This work is supported by the Young Talent Program of Beijing (YETP0770), the State Forestry Administration 948 Project (no. 2013-4-66), the Fundamental Research Funds for the Central Universities (no. 2015ZCQ-LY-01), and the National Natural Science Foundation of China (nos. 11371224 and 11001014).

\section{References}

[1] R. J. Gardner, Geometric Tomography, vol. 58 of Encyclopedia of Mathematics and Its Applications, Cambridge University Press, New York, NY, USA, 1995.

[2] R. J. Gardner and P. Gritzmann, "Discrete tomography: determination of finite sets by X-rays," Transactions of the American Mathematical Society, vol. 349, no. 6, pp. 2271-2295, 1997.

[3] G. T. Herman and A. Kuba, Discrete Tomography: Foundations, Algorithms, and Applications, Birkhäuser, Boston, Mass, USA, 1999.

[4] R. J. Gardner, P. Gronchi, and C. Zong, "Sums, projections, and sections of lattice sets, and the discrete covariogram," Discrete \& Computational Geometry, vol. 34, no. 3, pp. 391-409, 2005.

[5] J. Zhou, "On the projections of convex lattice sets," Acta Mathematica Sinica, vol. 26, no. 10, pp. 1969-1980, 2010.

[6] H. Xiong, "On a discrete version of Alexandrov's projection theorem," Acta Mathematica Sinica (English Series), vol. 29, no. 8, pp. 1597-1606, 2013.

[7] M. de Berg, O. Cheong, M. van Kreveld, and M. Overmars, Computational Geometry Algorithms and Applicationsy, Springer, Berlin, Germany, 2008. 


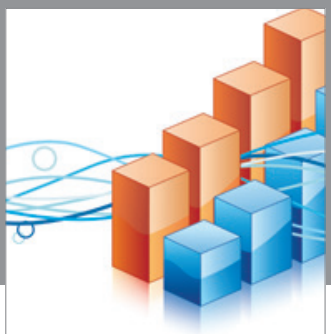

Advances in

Operations Research

vatem alat4

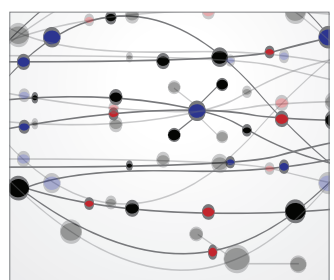

\section{The Scientific} World Journal
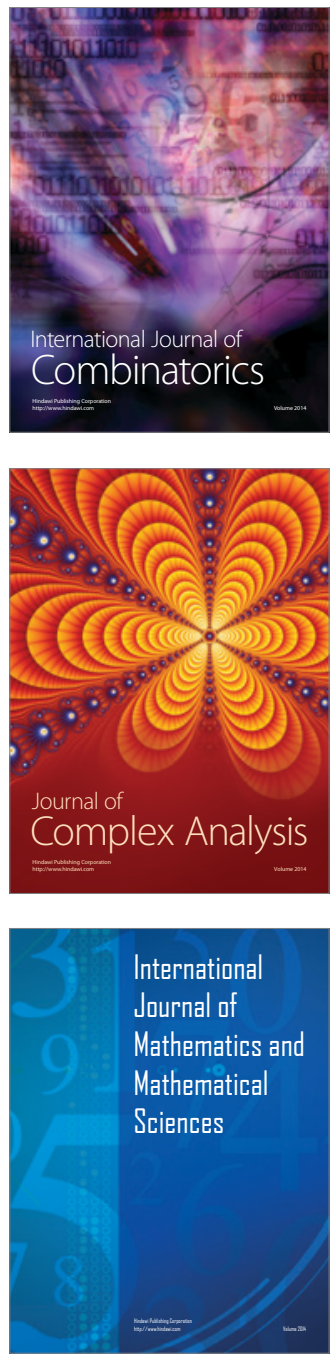
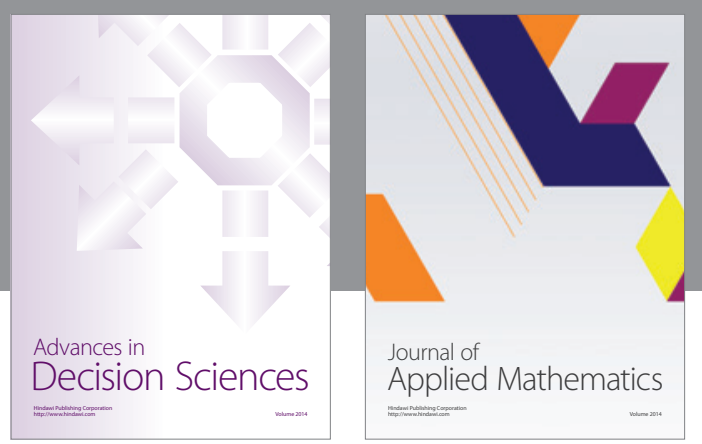

Algebra

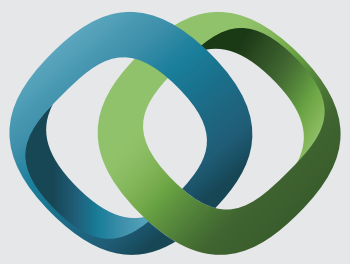

\section{Hindawi}

Submit your manuscripts at

http://www.hindawi.com
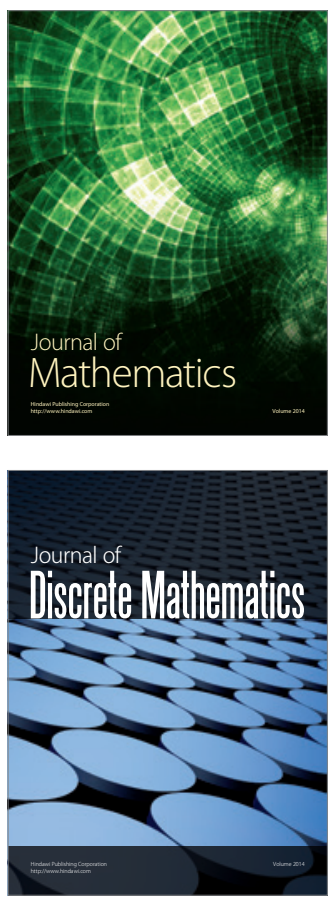

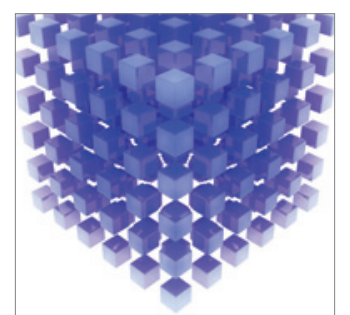

Mathematical Problems in Engineering
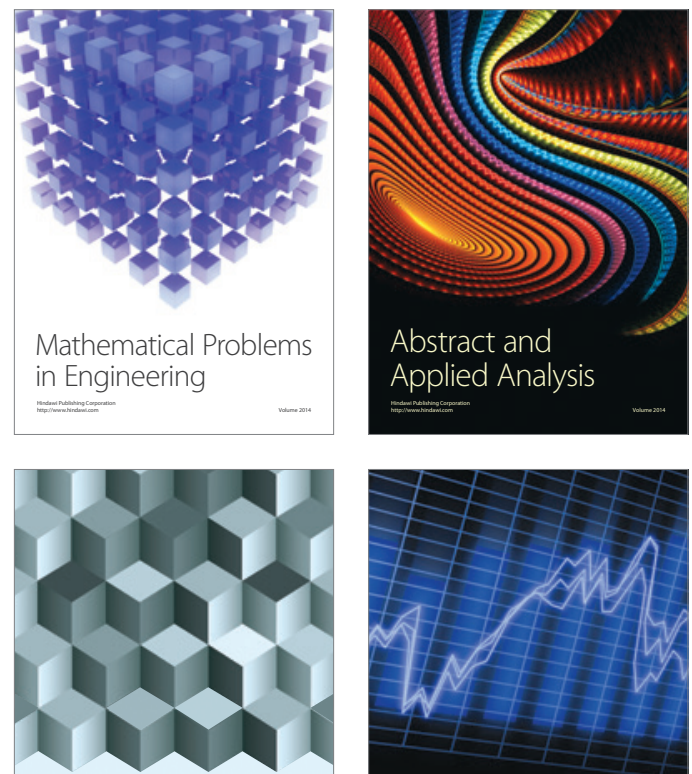

Journal of

Function Spaces

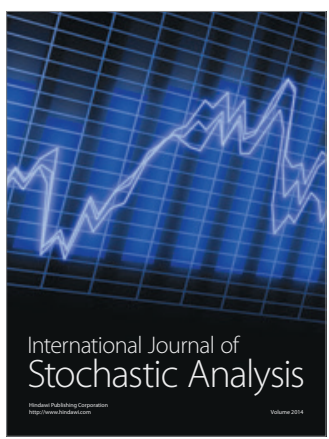

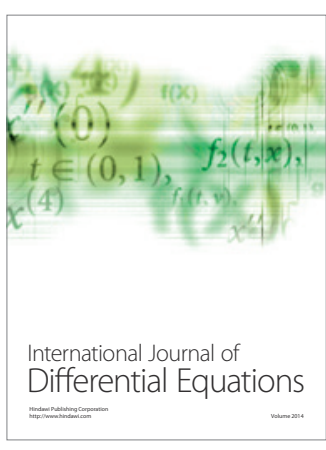
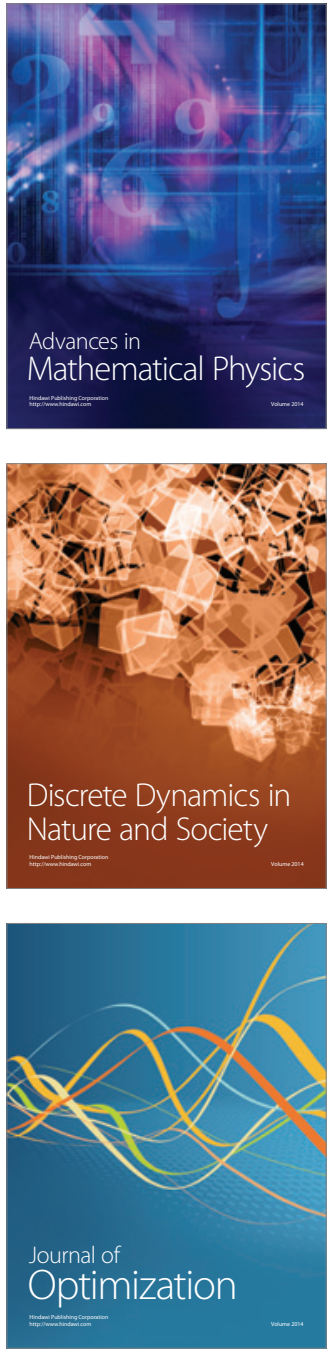